\title{
De la vivienda a la ciudad: Arquitectos de Cabecera, una propuesta alternativa de planificación bottom-up ${ }^{\prime}$
}

\author{
Elisabet Olivares Zapiain \\ URBES arquitectura y urbanismo, Perú
}

Recibido: 5 de septiembre del 2016 / Aprobado: 20 de diciembre del 2016

La experiencia académica Arquitectos de Cabecera, que nació en la Escuela de Arquitectura de la Universidad Politécnica de Cataluña y que posteriormente desarrolló una sección en colaboración con el posgrado Procesos Urbanos y Ambientales [URBAM-EAFIT] de Medellín, ha trabajado diversos casos, cuyo análisis permite imaginar la construcción de nuevas cartografias de la ciudad partiendo desde lo local. A modo de una estrategia complementaria, el análisis local, nos permite tener una visión alternativa, partiendo de los problemas de sus ciudadanos, cartografiándolos en un proceso ascendente, desde lo micro (la vivienda) hasta lo macro (la ciudad). Un proceso de ida y vuelta, donde se reconoce lo pequeño como unidad de agregación de la gran ciudad. La vivienda, genérica y anónima, constituyente de las ciudades.

vivienda, urbanismo bottom-up, actores locales, cartografías urbanas

\section{From the Dwelling to the City: "Arquitectos de Cabecera", an Alternative to Bottom-Up Planning}

The academic experience "Arquitectos de Cabecera", born in the School of Architecture at Universitat Politècnica de Catalunya with one section later developed in collaboration with Medellin "Urban and Environmental Processes" [URBAM-EAFIT] Graduate school, has worked on different cases trying, it is possible to imagine the design of new city maps from a local point of view. As a complementary strategy, the local analysis, detailed case by case, provides an alternative view for professionals to recognize bit by bit the specific problems of the cities based on the problems of their citizens, which would let them create a new bottom-up cartography: from micro (housing) to macro (city). A round-trip process, where the "small part" is accepted as an adding unit of the city. The housing is a general and unknown element, a majority and constituent part of the cities.

housing, bottom up urbanism, local actors, urban cartographies

1 Artículo de análisis de un proyecto académico realizado en la Maestría de Procesos Urbanos y Ambientales URBAM-EAFIT, Medellín, Colombia (marzo del 2016). 


\section{INTRODUCCIÓN}

El proyecto surge en los pasillos de la Escuela Técnica Superior de Arquitectura de Barcelona (ETSAB) de la Universidad Politécnica de Cataluña (UPC), en el contexto de la asignatura Proyectos III-IVVivienda y Ciudad, a cargo del arquitecto Pere Joan Ravetllat, como un mecanismo de análisis de la realidad concreta de los lugares y sus habitantes. Los estudiantes visitan barrios como el Raval o Bellvitge y buscan, conversando con los habitantes del barrio, soluciones concretas y de pequeña escala para mejorar la calidad de vida de los vecinos.

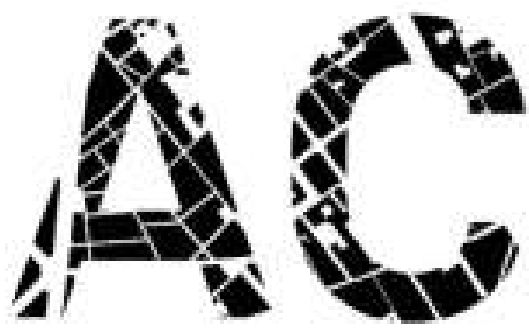

\section{ARQUITECTOS DE CABECERA}

Figura 1

Logo de Arquitectos de Cabecera

Fuente: https://www.facebook.com/arquitectosdecabecera/?fref=ts

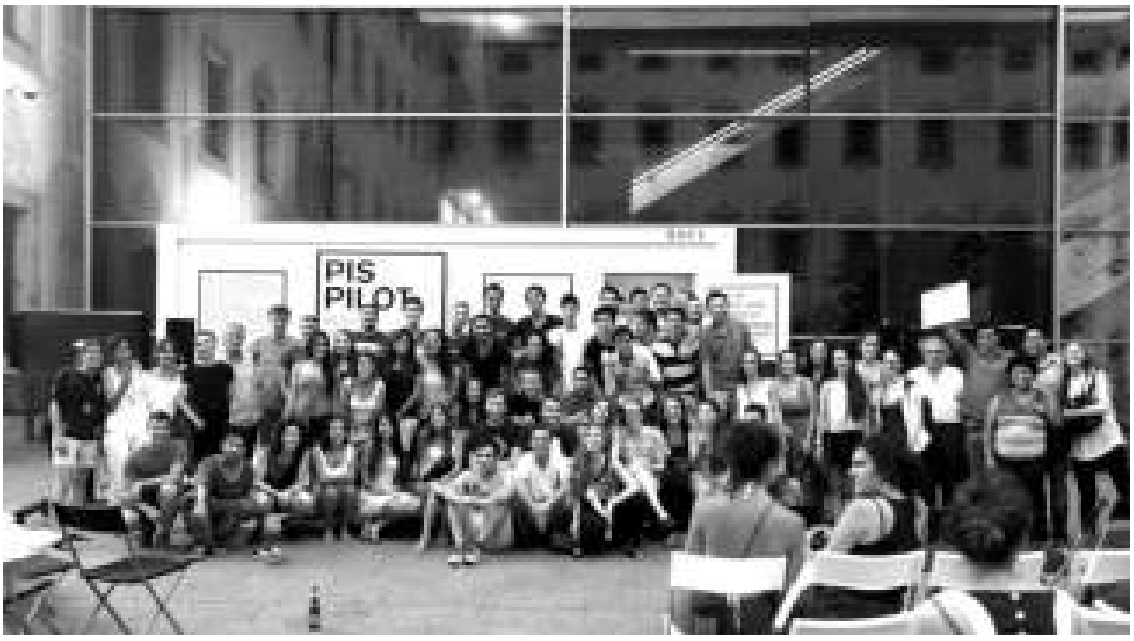

Figura 2

Equipo participante en Arquitectos de Cabecera ETSAB

Fuente: https://www.facebook.com/arquitectosdecabecera/?fref=ts 
La imagen macro e institucional de una ciudad como Barcelona, reconocida en todo el mundo como un modelo de desarrollo urbano, pudiera hacernos pensar que los aspectos más básicos del hábitat, como la vivienda digna y accesible, debieran estar cubiertos en su totalidad. Sin embargo, la precariedad puertas adentro manifiesta en los barrios estudiados pone sobre el tapete problemas de estructura derivados de un desarrollo poco equitativo de la ciudad, que ha generado bolsas urbanas de precariedad en un contexto agudizado por la crisis económica, donde la vivienda ha sido un aspecto especialmente maltratado.

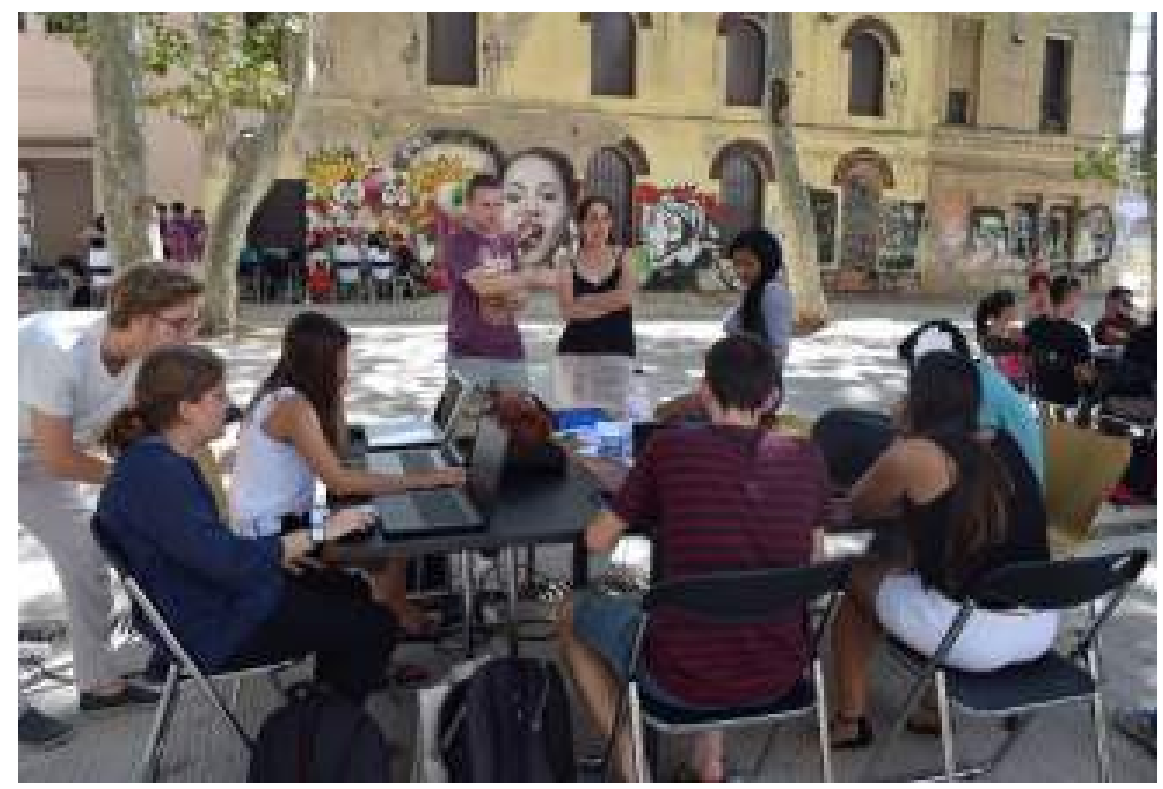

Figura 3

Proceso y desarrollo del taller

Fuente: https://www.facebook.com/arquitectosdecabecera/?fref=ts 
Avquilecto de cabecera presenta

No todos los

futuros para Poblenou

son iguales.

Ven para elegir el

que nos conviene.

N23. Atupclew Hpassaigcalnel

${ }^{\circ} \mathrm{c}$

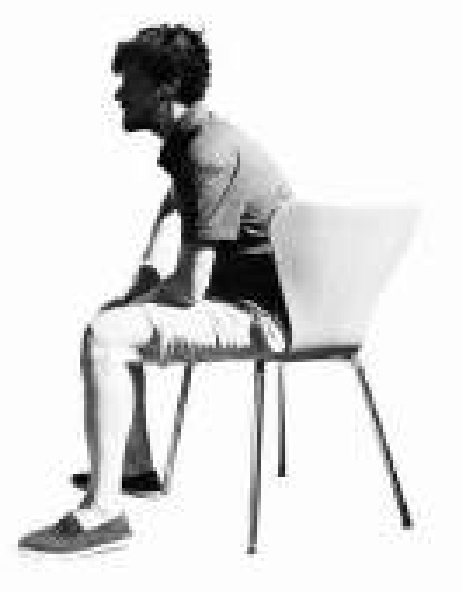

Figura 4

Llamado al proceso participativo del taller

Fuente: https://www.facebook.com/arquitectosdecabecera/?fref=ts

Dicho esto, los principales objetivos del programa original son los siguientes:

- Reconsiderar el rol contemporáneo del arquitecto y su utilidad como técnico al servicio del ciudadano.

- Recuperar la importancia del usuario en el proceso del diseño participativo.

- Romper la barrera entre la academia y la sociedad, fomentando la proximidad entre estudiantes y usuarios.

La consolidación del programa como propuesta académica, al cual las autoridades municipales han otorgado recientemente el Premio Ciudad de Barcelona, ha acompañado el desarrollo de la experiencia en otros ámbitos mediante la Maestría de Procesos Urbanos y Ambientales-URBAM-EAFIT. El caso que nos ocupa es la ciudad de Medellín, un contexto urbano donde las autoridades y los técnicos han logrado grandes avances en la mejora de la calidad de vida, gracias a la introducción estratégica de equipamientos, espacios públicos y conectividad metropolitana. Sin embargo, la vivienda 
social sigue siendo un problema complejo y de gran escala, motivo de constante debate e investigación.

A pesar de conservar la esencia del problema, se evidencia el cambio drástico de contexto: de una ciudad como Barcelona, construida en un marco de amplia institucionalidad, larga trayectoria y experiencia en grandes proyectos de mejora y transformación urbana, a una ciudad latinoamericana emergente, donde la parte mayoritaria se viene consolidando mediante procesos informales y autoconstruidos. A la lectura inicial, donde la propuesta se articulaba en torno a la vivienda como eje central de la investigación y al contacto directo entre arquitecto y usuario en intervenciones a pequeña escala, se añade la necesidad de una lectura urbana donde la vivienda ha desempeñado un papel esencial como unidad de agregación espontánea en los tejidos urbanos a escala metropolitana.

La ciudad vista desde arriba (el dron) vs. la ciudad vista desde abajo (el peatón) y el caso específico de las ciudades emergentes: el encuentro entre la planificación macroinstitucional y la microplanificación popular

Desde el origen del urbanismo como disciplina, la mirada holística y a gran escala de las ciudades ha permitido investigar y comprender aspectos de carácter macro y territorial, mediante los cuales ha sido posible proponer redes, sistemas e infraestructuras, a fin de buscar soluciones efectivas y solventes, que atendiera las distintas necesidades que requería la ciudad. Esta mirada desde arriba ha venido acompañada en la práctica urbanística de una actitud y unas estrategias de intervención descendentes, es decir, la iniciativa y las estrategias las decidían las grandes instituciones y luego eran comunicadas a los ciudadanos para su validación.

La mirada estratégica metropolitana, responsable de la toma de decisiones macro y de una cartera de proyectos que estructuren la complejidad de una ciudad, es indiscutiblemente necesaria para la articulación general. Sin embargo, considerarla como único punto de partida derivaría en diagnósticos abstractos, lejanos y difíciles de aplicar. De forma paralela, en la práctica profesional han empezado a proliferar proyectos de intervención urbana que reivindican una mirada alternativa conocida como bottom-up o ascendente, gracias a la cual los procesos participativos nacidos a través de acuerdos a 
pequeña escala entre los vecinos o actores locales se materializan en proyectos urbanos de carácter, hasta la fecha, eminentemente barrial.

La complementariedad entre estas dos visiones, estrategias ascendentes y descendentes, para concertar proyectos de validación y consenso entre la ciudadanía y las instituciones, es fundamental a la hora de incorporar nuevos actores y enfoques a la construcción colectiva de la ciudad. Y lo es todavía con mayor razón en el contexto de las ciudades emergentes latinoamericanas, donde la unidad de vivienda popular ha asumido históricamente un papel estratégico en la construcción de la ciudad, como un agregado progresivo de tejidos informales. En resumen, ciudades cuyo ADN registra la esencia de los fenómenos y los procesos ascendentes de consenso y participación.

La consideración e incorporación de esta mirada complementaria desde abajo es estratégica para comprender de manera cabal la complejidad de los procesos urbanos actuales. A partir de dicha mirada complementaria y desde lo local, se enmarca la propuesta pedagógica de Arquitectos de Cabecera desarrollada en Medellín. En esta ciudad, un análisis pormenorizado de una unidad de vivienda y su contexto permite identificar problemas existentes en el barrio y en la ciudad, que, sin embargo, pueden no haber sido detectados en la mirada macro de la planificación convencional o, al menos, no en toda su complejidad.

En este trabajo de ida y vuelta, lo descubierto a escala micro puede y debe ser incorporado en un marco más amplio que permita, una vez analizados desde una perspectiva holística metropolitana, volver a descender para comprobar su integración y respuesta en la escala micro de la que partió, y así sucesivamente hasta lograr el balance necesario entre actores, escalas, estrategias y enfoques.

Por lo general, el urbanismo dual de la vivienda a la ciudad (ascendente) y de la ciudad a la vivienda (descendente) todavía no ha sido incorporado en las estrategias institucionales, donde haya miradas alternativas de actores diversos que contribuyan a una lectura integral y complementaria de la ciudad en toda su complejidad. 

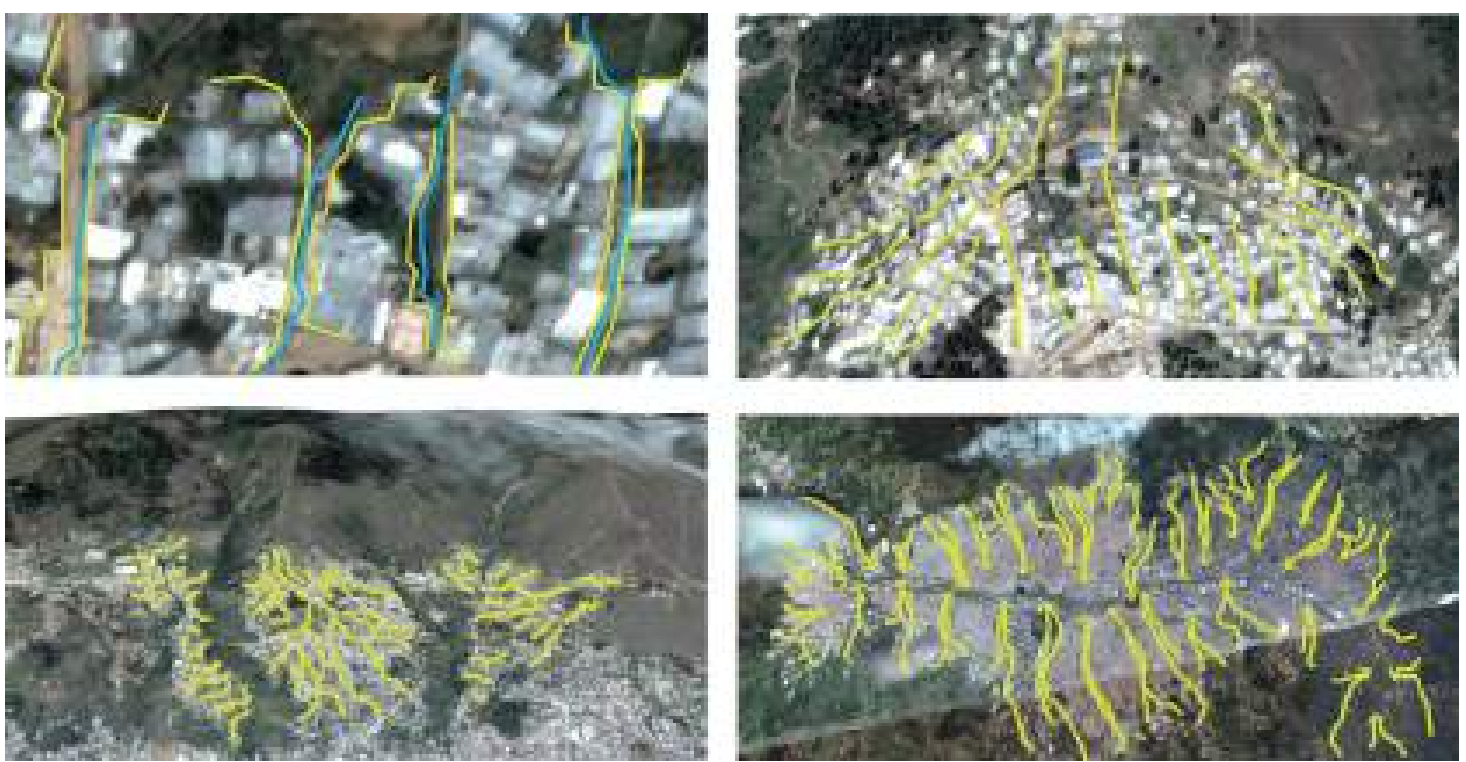

Figuras $5,6,7$ y 8

Identificación a distintas escalas de un problema y una posibilidad derivados del análisis de la escala local: lectura dual y complementaria de la ciudad; de la ausencia de canalización de las aguas residuales en la vivienda a la incorporación de las quebradas naturales como espacios públicos metropolitanos (grupo de trabajo de la familia Luz Delbi [Juan Fernando Zapata, Janeth Arango, Paola Ramos, Carlos Montoya, Elisabet Olivares])

\section{METODOLOGÍA Y DESARROLLO DEL EJERCICIO}

Durante cuatro días y a modo de laboratorio inicial, los estudiantes de posgrado, en grupos de cuatro o cinco personas conformados por técnicos y profesionales de diversas disciplinas (arquitectos, abogados, técnicos agrícolas, biólogos, sociólogos, politólogos, ingenieros civiles) cuestionaron paradigmas y formularon preguntas. Durante el ejercicio, se buscó una mirada interdisciplinar con la cual los distintos aportes lograran identificar, abordar y proponer una solución, en colaboración con las familias, en tan corto tiempo.

El escenario escogido para el desarrollo del ejercicio fue el borde urbanorural de la ciudad de Medellín, donde la ocupación informal en ladera, fruto 
de las migraciones del campo a la ciudad, derivadas principalmente del conflicto armado en el país y de la incapacidad del estado de garantizar el acceso a la vivienda, han desencadenado actualmente importantes conflictos urbanos, sociales y ambientales. A la fecha y según cifras de la Secretaría de Planeación metropolitana, se estima que en una ciudad intermedia como Medellín más de 27000 viviendas se encuentran localizadas en áreas de alto riesgo no mitigable en este sector ( ${ }^{*}$ POTMedellín2013) y que, según los índices y las proyecciones, esta cifra aumenta constantemente, sin existir propuestas de mitigación o mejora. Se ha elegido esta ciudad por ser un contexto estratégico, donde es altamente necesaria la búsqueda de soluciones alternativas para garantizar un hábitat seguro a los pobladores, reducir el riesgo y controlar las pérdidas medioambientales derivadas de la mala y descontrolada ocupación de suelo.
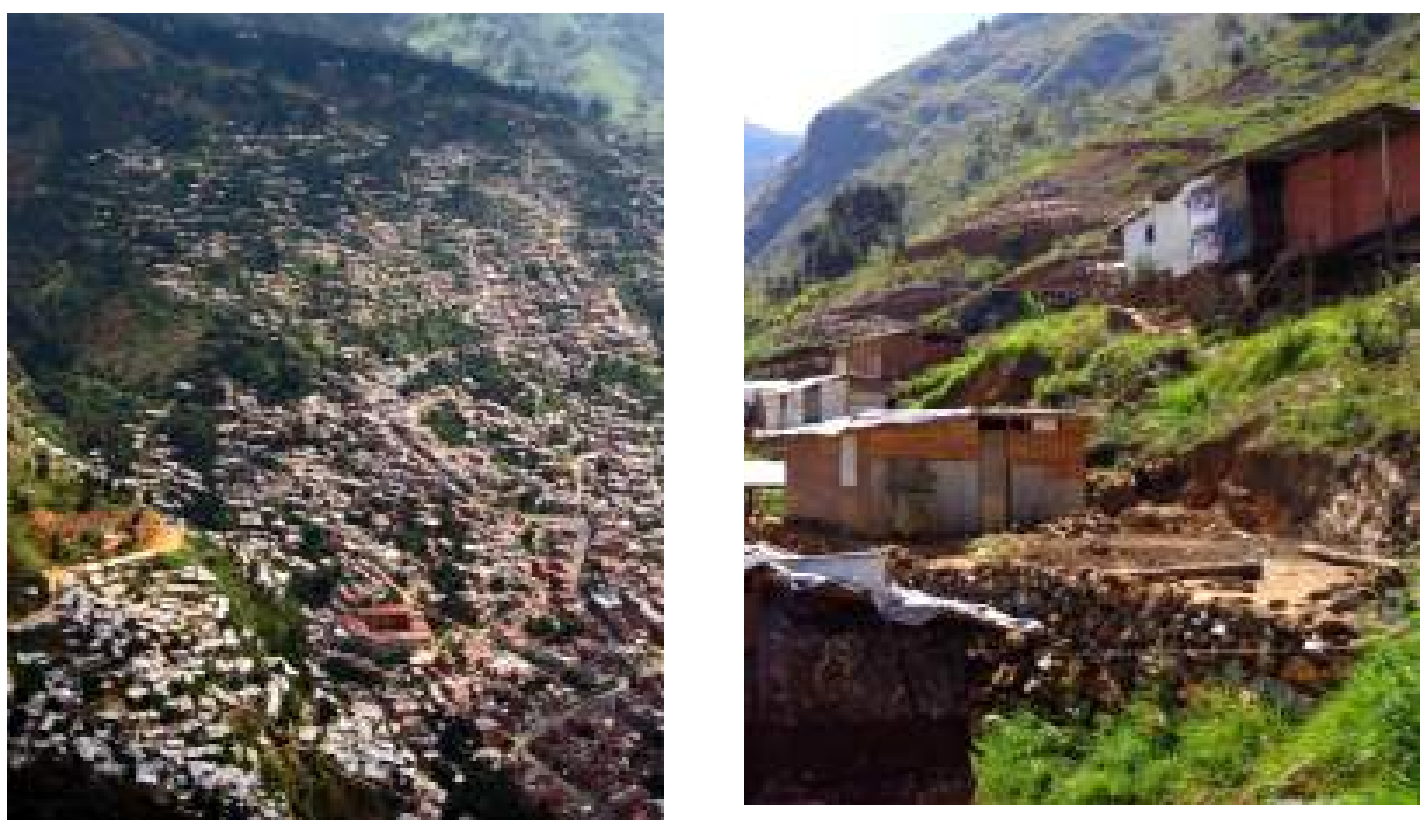

Figuras 9 y 10

Distintas aproximaciones al ámbito de estudio (barrio La Cruz, sector La Honda, ladera nororiental).

Fuente: Rehabitar la montaña. Estrategias y procesos para un hábitat sostenible en las laderas de Medellín de URBAM EAFIT 
Se había tomado contacto con las familias mediante organizaciones activas de la sociedad civil reconocidas por actuar estratégicamente con las familias en el ámbito de estudio (barrio La Cruz, sector La Honda, en la ladera nororiental de la ciudad). Estas asociaciones y lazos preexistentes contribuyeron en gran medida a establecer una relación de confianza entre técnicos y familias, quienes abrieron no solo sus viviendas, sino que compartieron también con todo el equipo problemas personales, necesidades y aspiraciones familiares, profesionales o económicas.

El encuentro se convirtió en una ventana a la identificación y priorización de pequeñas y medianas intervenciones que, a modo de catalizadores, pudieran contribuir a la mejora de su calidad de vida y desencadenar procesos sociales y económicos que a mediano plazo supusieran un punto de inflexión: modelos de negocios participativos, ampliaciones estratégicas en la vivienda o mejoras en una precaria red de saneamiento. Cada familia pudo manifestar y mostrar para su análisis los problemas que consideraban prioritarios, pero también sus sueños y aspiraciones de progreso y mejora.Y todo ello desde su vivienda.

Las conversaciones y el levantamiento estratégico y consensuado de información hacían énfasis en las principales preocupaciones de cada familia. Se hizo un reconocimiento de la infraestructura, la habitabilidad, los ingresos familiares y la estructura familiar. Cada caso aportaba mapeos y miradas específicas de acuerdo a los síntomas reconocidos en él.

La lluvia de ideas inicial fue la principal fuente de información para las sesiones técnicas sucesivas, donde el intercambio de puntos de vista procedentes de diversas disciplinas trataban de acotar los escenarios, priorizar las alternativas y diseñar propuestas técnicas capaces de resolver los restos previstos. Desde el reconocimiento de la vivienda hasta una visión intermedia de su contexto en el barrio, tanto en el aspecto urbano como en el social, se elaboró un mapa de actores locales — asociaciones, comadres, líderes comunitarioscon quienes construir escenarios a mediano y largo plazo. 


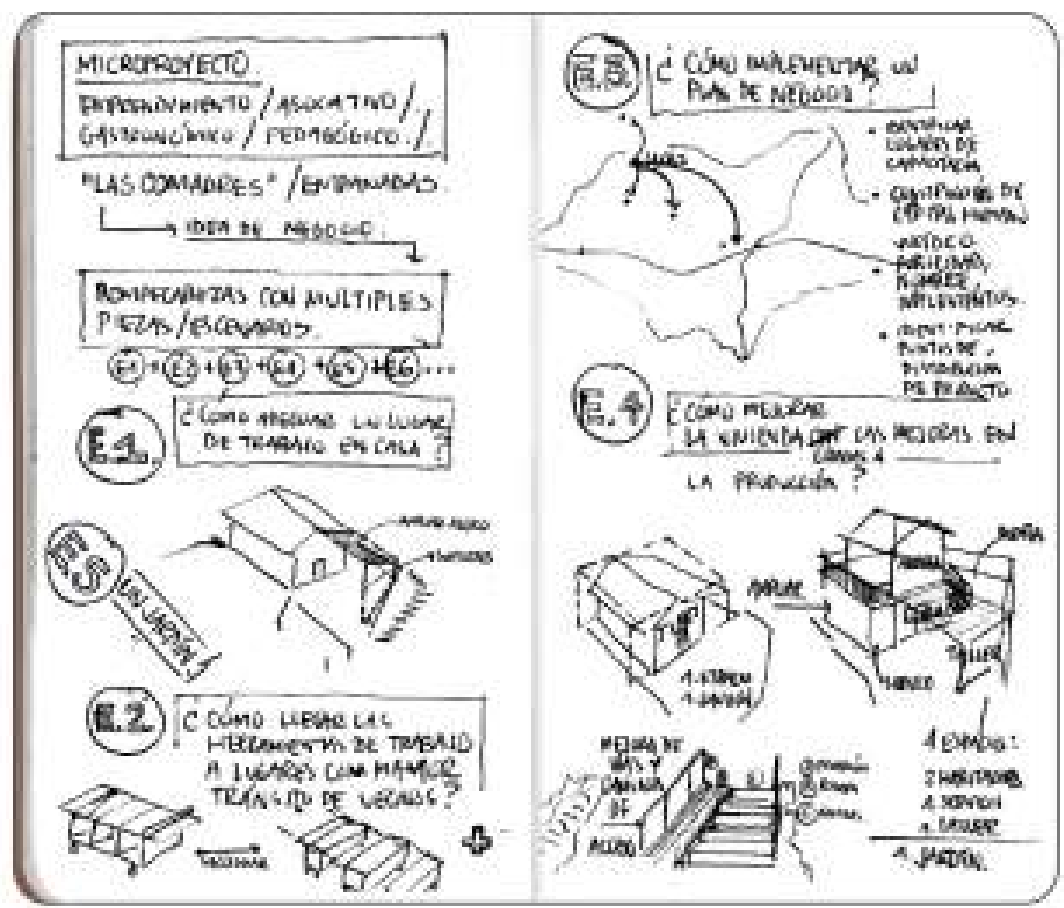

Figura 11

Levantamiento gráfico de ideas surgidas de la conversación con los usuarios

Con las propuestas técnicas se desarrollaron estrategias sociales, legales y económicas en busca de posibilidades que permitieran a los vecinos poner en marcha las estrategias de desarrollo de la propuesta. 


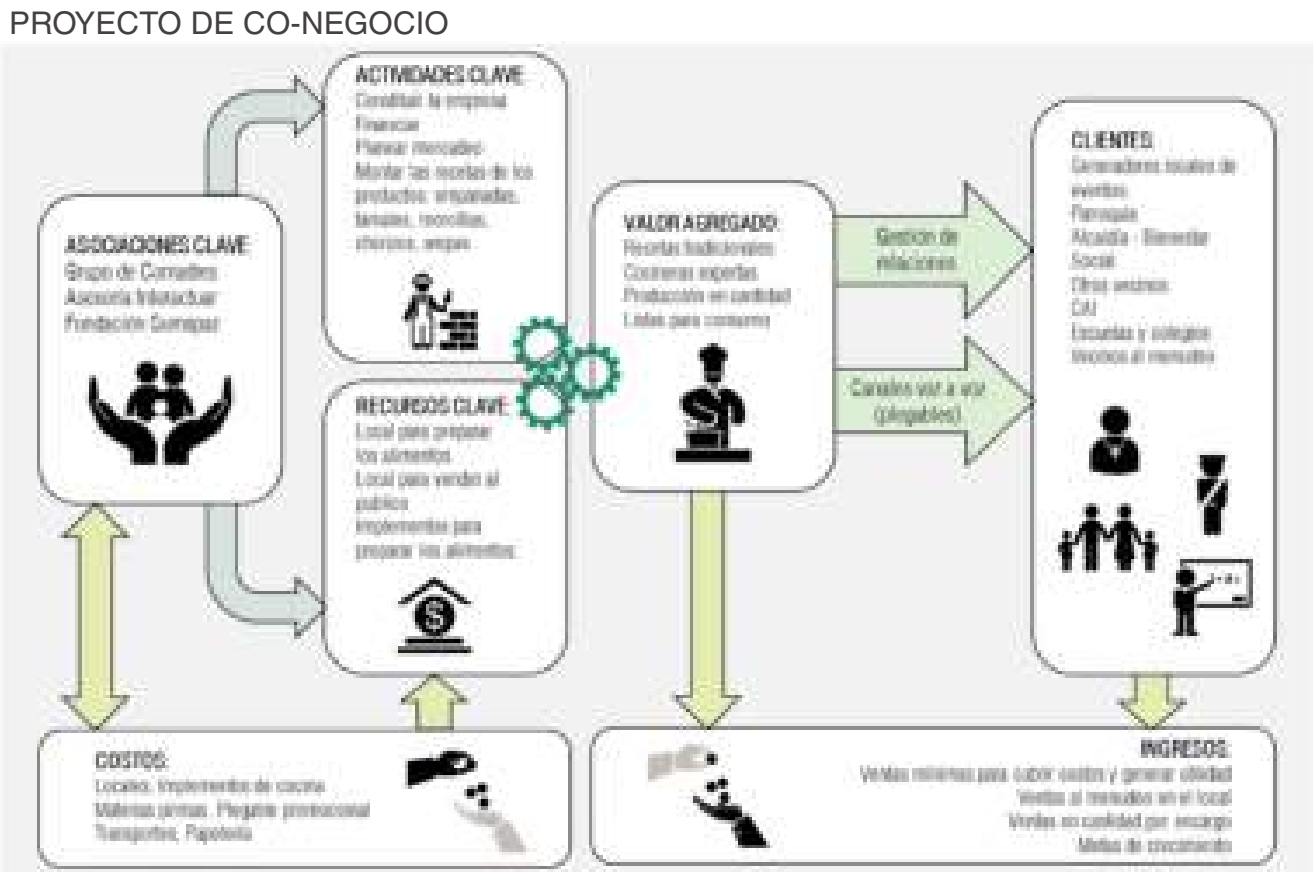

Figura 12

Propuesta inicial para iniciar un negocio participativo entre los actores de la comunidad

Fuente: Grupo de trabajo: familia de doña Graciela (Andrea Cifuentes, Fernando Cortés, Sebastián Serna, Adolfo León Tabares)

\section{RESULTADOS}

Luego de estos días de trabajo, las familias celebraron una última sesión en la que manifestaron sus desencuentros, dificultades, problemas, posibilidades y resultados. Esta sesión de trabajo fue también una llamada a la reflexión sobre la experiencia académica, personal y profesional, y un punto seguido en la continuación de cada uno de los microproyectos planteados en el proceso. 


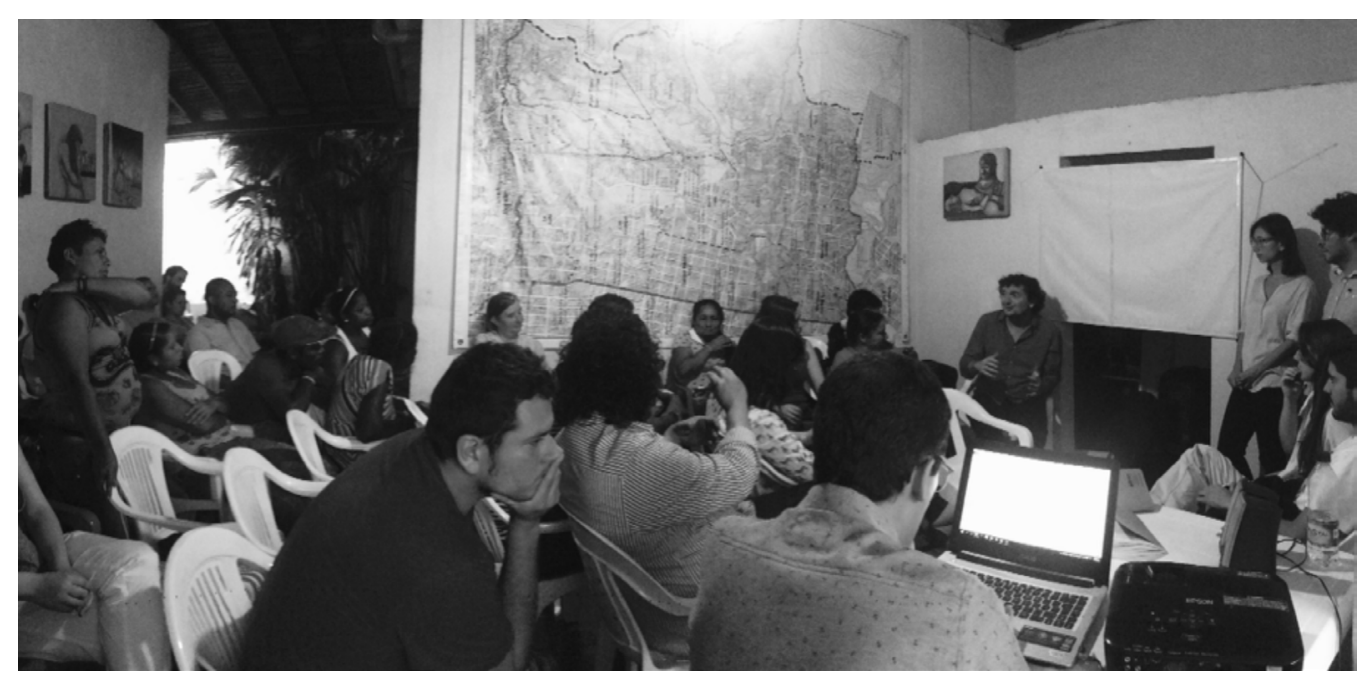

Figura 13

Sesión final de trabajo, intercambio de ideas entre familias, miembros de la comunidad, técnicos y profesionales

\section{CONCLUSIÓN}

El enfoque de estos ensayos académicos genera nuevas preguntas relacionadas con la planificación urbana de unas ciudades emergentes, complejas y ricas en dinámicas, donde la vivienda y la pequeña escala asociativa asume un papel protagónico en la conformación de la gran ciudad metropolitana.

¿Es posible incorporar procesos de ida y vuelta en la lectura de la ciudad donde se recuperen fenómenos de pequeña escala y contribuyan a la lectura global? ¿Cómo integrar estos procesos participativos en la planificación institucional de una manera efectiva para el proceso y de una manera atractiva para los ciudadanos? ¿Hasta qué punto es posible encontrar un equilibrio entre los proyectos marco de validación y los proyectos participativos de consenso? ¿Debe existir esa categorización? ¿Puede la vivienda (micro) aportar pistas sobre los procesos urbanos (macro)? 
La experiencia abrió posibilidades de plantear nuevas alternativas académicas y profesionales de aproximación a la ciudad desde una lectura ascendente, donde el análisis pormenorizado de la vivienda como unidad de agregación, en constante cambio y adaptación dentro de un tejido en proceso de consolidación, puede aportar nuevas ideas y enfoques al diagnóstico y propuesta macro de una planificación a mayor escala en un trabajo doble ascendente y descendente.

Del mismo modo y a pesar del corto periodo de tiempo, el ejercicio contribuyó a romper ideas preestablecidas sobre el rol del arquitectourbanista y, en general, profesionales vinculados al desarrollo de planificación de la ciudad, en la sociedad actual, apostando por un desarrollo participativo en un proceso de planificación urbana que recupera la importancia del usuario en el diseño.

\section{BIBLIOGRAFÍA}

Borja, J. (Marzo de 2012). Revolución urbana y derechos ciudadanos. Claves para interpretar las contradicciones de la ciudad actual (tesis doctoral). Barcelona: Universitat de Barcelona.

Cárdenas, J. C., Maya, D. L., y Lopez, M. C. (2003). Métodos experimentales y participativos para el análisis de la acción colectiva y la cooperación en el uso de recursos naturales por parte de comunidades rurales. Cuadernos de Desarrollo Rural, 96-63.

Castro, M., y Martí, M. (2016). Comunes urbanos: de la gestión colectiva al derecho a la ciudad. Eure, 42(125), 131-153.

Eslava, A. (2017). Políticos, técnicos y comunidades. Medellín: Universidad EAFIT.

Foro Social Mundial, Foro Urbano Mundial. (2001). ONUHabitat. Recuperado de http://www.onuhabitat.org/index.php?option=com_ docman\&task $=$ doc_details\&gid $=50 \&$ Itemid $=3$

Harvey, D. (2013). Ciudades rebeldes. Del derecho a la ciudad a la revolución urbana. Salamanca: Akal. 
Ibáñez, R., y Castro, C. (2015). Los comunes en perspectiva: eficiencia versus emancipación. En Economistas sin Fronteras. Dossieres, 16, 8-12.

Lefebvre, H. (1968). El derecho a la ciudad. Barcelona: Península.

Naciones Unidas.Asamblea General.(2016). Documento de política 1: derecho a la ciudad y ciudades para todos. Surabaya (Indonesia): Naciones Unidas.

Ostrom, E. (2000). El gobierno de los bienes comunes. La evolución de las instituciones de acción colectiva. México: Fondo de Cultura Económica. 